\title{
DOMINATED EXTENSIONS OF FUNCTIONALS AND $V$-CONVEX FUNCTIONS ON CANCELLATIVE CONES
}

\author{
S. Romaguera, E.A. Sánchez Pérez and O. Valero
}

\begin{abstract}
Let $C$ be a cancellative cone and consider a subcone $C_{0}$ of $C$. We study the natural problem of obtaining conditions on a non negative homogeneous function $\phi: C \rightarrow R^{+}$ so that for each linear functional $f$ defined in $C_{0}$ which is bounded by $\phi$, there exists a linear extension to $C$. In order to do this we assume several geometric conditions for cones related to the existence of special algebraic basis of the linear span of these cones.
\end{abstract}

\section{INTRODUCTION}

Let $R$ be the field of real numbers and let $R^{+}$be the set of non-negative real numbers. A cone $C$ is a triple $(C,+,$.$) such that (C,+)$ is a monoid and the product . : $R^{+} \times C \rightarrow C$ satisfies the usual axioms of a linear space when restricted to non negative scalars. A subset $B$ of a real linear space $X$ is said to be algebraically closed when $a+b \in B$ and $\lambda a \in B$ for each pair $a, b \in B$ and $\lambda \in R^{+}$. It can be proved that each cancellative cone can be identified with an algebraically closed subset of a linear space (see [2, 11]). A non negative function $\phi$ defined on a cone $C$ is called positively homogeneous if $\phi(a x)$ $=a \phi(x)$ for every $x \in C$ and $a \in R^{+}$. Such a function is convex provided that $\phi(x+y)$ $\leqslant \phi(x)+\phi(y), x, y \in C$. In [6] the reader can find several results related to extensions of such kind of functions defined from cancellative cones to linear spaces.

It is possible to find a lot of dominating extension results for functionals defined on cones in the bibliography (see for example $[2,11,1]$ ). However, these results depend on particular properties of the dominating function $\phi$, for which is usual to require convexity. Arguments related to order relations defined in the cones are also used (see [13]). Moreover, the results are often obtained for extended functions $\phi$ (that is, functions $\phi$ defined in the extended non negative real line $R^{+} \cup\{\infty\}$ ). In this paper we deal with cancellative cones that satisfy a geometric condition related to certain generating properties of algebraic basis of the linear space that contains the cone. We obtain those conditions

Received 13th May, 2002

The authors acknowledge the support of the Spanish Ministry of Science and Technology, under grant BFM2000-1111, and the support of the Universidad Politécnica de Valencia, under grant 20000592 for Interdisciplinary Research Projects.

Copyright Clearance Centre, Inc. Serial-fee code: 0004-9727/03 \$A2.00+0.00. 
that a positively homogeneous real function $\phi$ defined on a cancellative cone $C$ must have so that each linear function defined on a subcone $C_{0}$ and dominated by $\phi$ has a linear extension to the whole $C$.

Our notation is standard. The natural numbers will be denoted by $N$. The main references about the Hahn-Banach Theorem for linear spaces and cones are $[12,2,11]$. If $B$ is a subset of a cone $C$, we define $\operatorname{span}\{B\}$ as the set of positive linear combinations of elements of $B$, that is, the elements of $\operatorname{span}\{B\}$ would be written as $\lambda_{1} x_{1}+\cdots+\lambda_{n} x_{n}$, where $n$ is a natural number, $\lambda_{i} \in R^{+}$and $x_{i} \in B$ for every $i=1, \ldots, n$. It is obvious that $\operatorname{span}\{B\}$ is a subcone of $C$. Since $C$ is a subset of a linear space $X$, we can also consider the linear subspace linspan $\{B\}$ of $X$ defined as the (non necessarily positive) linear combinations of elements of $B$. If $C$ is a cancellative cone and $\mathcal{B}$ is a basis of linspan $\{C\}$ we shall simply say that $\mathcal{B}$ is a basis of $C$. Thus, we shall say that the dimension of a cone $C$ is $n$ if the dimension of linspan $\{C\}$ is $n, n \in N \cup\{\infty\}$.

DEFINITION 1: Let $C$ be a cancellative cone and let $C_{0}$ be a subcone of $C$. We say that $C_{0}$ is compatible with an algebraic basis $V=\left\{v_{i}: i \in I\right\}$ of linspan $\{C\}(V$ compatible for short) if there is a subset $J$ of $I$ such that $V_{0}=\left\{v_{i}: i \in J\right\}$ is an algebraic basis of linspan $\left\{C_{0}\right\}$.

It is well known that each linear space $X$ has an algebraic basis. Moreover, if $W$ is a basis for a subspace $S$ of $X$, it can be always found a basis $V$ for $X$ such that $W \subset V$ (see [8, Theorem 2.4, Chapter IV]). This means that for each subcone $C_{0}$ of a cancellative cone $C$ there exists always a basis $V$ of $C$ such that $C_{0}$ is $V$-compatible.

DEFINITION 2: We say that a cancellative cone $C$ is well generated by an algebraic basis $V=\left\{v_{i}: i \in I\right\}$ of linspan $\{C\}$ if for every subset $J$ of $I$ and each $i_{0} \in I-J$, we can find for every element

$$
y \in \operatorname{linspan}\left\{\left\{v_{i}: i \in J\right\}, v_{i_{0}}\right\} \cap C,
$$

a representation $y=x+\lambda v_{i_{0}}$, where $x \in C \cap \operatorname{linspan}\left\{v_{i}: i \in J\right\}$ and $\lambda \in R$.

It is easy to find examples of well generated cones by algebraic basis. For instance, every linear space is well generated by any algebraic basis. Another easy example is the positive cone of $R^{n}$, that is well generated by the canonical basis of $R^{n}$. However, we can find examples of cones that are not well generated by particular basis. For instance, consider the positive cone $\left(R^{3}\right)^{+}$of $R^{3}$ and the basis given by the set of vectors $B=\{(1,1,0),(1,0,1),(0,0,1)\}$, where $(x, y, z)$ are the coordinates with respect to the canonical basis. The element $(0,1,0)$ of the positive cone of $R^{3}$ can not be written as a sum of an element of linspan $\{(1,1,0),(1,0,1)\} \cap\left(R^{3}\right)^{+}$and an element of linspan $\{(0,0,1)\}$.

\section{2. $V$-CONVEXITY AND DOMINATED EXTENSIONS OF FUNCTIONALS.}

DEFINITION 3: Let $C$ be a cancellative cone. Consider an algebraic basis $V=\left\{v_{i}\right.$ : $i \in I\}$ of $C$. Let $C_{0}$ be a $V$-compatible subcone of $C$ with a basis $V_{0}=\left\{v_{i}: i \in J\right\}$, 
$J \subset I, J \neq I$. We say that a positively homogeneous function $\phi$ is $V$-convex with respect to $C_{0}$ if for every $i \in I-J$ and $x, y \in C_{0}$ such that $x+v_{i}, y-v_{i} \in C$,

$$
\phi(x+y) \leqslant \phi\left(x+v_{i}\right)+\phi\left(y-v_{i}\right) .
$$

It is easy to see that convexity for the function $\phi$ implies $V$-convexity with respect to every $V$-compatible subcone $C_{0}$ and each algebraic basis $V$. The following example shows that the converse is not true, that is, we can find a $V$-convex function with respect to a one dimensional subcone of a semiplane of $R^{2}$ that is not convex.

EXAmple 4: Consider the canonical basis $\mathcal{B}=\left\{e_{1}, e_{2}\right\}$ of $R^{2}$ and the cone $C$ $=\operatorname{span}\left\{e_{1}, e_{2},-e_{2}\right\}$. We can consider the subcone $C_{0}=\operatorname{span}\left\{e_{1}\right\}$ and the function $\phi_{0}$ given by

$$
\phi_{0}(z)=\lambda+|\mu|, \quad z=\lambda e_{1}+\mu e_{2}, \quad \lambda>0, \mu \in R,
$$

and $\phi_{0}\left(\lambda e_{2}\right)=\phi_{0}\left(-\lambda e_{2}\right)=0$ for every $\lambda \in R^{+}$.

This function is not convex, since $\phi_{0}\left(e_{1}+e_{2}\right)=2$ but $\phi_{0}\left(e_{1}\right)=1$ and $\phi_{0}\left(e_{2}\right)=0$. However, it is $\mathcal{B}$-convex with respect to $C_{0}$, since for each $\lambda_{1}, \lambda_{2}>0$,

$$
\phi_{0}\left(\lambda_{1} e_{1}+\lambda_{2} e_{1}\right)=\lambda_{1}+\lambda_{2}<\lambda_{1}+\lambda_{2}+2=\phi_{0}\left(\lambda_{1} e_{1}+e_{2}\right)+\phi_{0}\left(\lambda_{2} e_{1}-e_{2}\right),
$$

and in the case that $\lambda_{1}=0$ or $\lambda_{2}=0$ the inequalities also hold.

DEFINITION 5: We say that a cone $C$ has the 1-extension property with respect to $\phi$ if for each one dimensional subcone $D$ of $C$ and each linear function $f: D \rightarrow R$ such that $f \leqslant \phi$ on $D$ there is a linear extension $\bar{f}$ to $C$ that satisfies $\bar{f}(x) \leqslant \phi(x)$ for every $x \in C$.

THEOREM 6. Let $C$ be a cancellative cone. Consider a positively homogeneous function $\phi: C \rightarrow R^{+}$. If $C$ has the 1-extension property with respect to $\phi$, then for every algebraic basis $V$ of $C$ and every $V$-compatible subcone $C_{0}, \phi$ is $V$-convex with respect to $C_{0}$.

Proof: Let $V$ be an algebraic basis of $C$. Suppose that $\phi$ is not $V$-convex with respect to the $V$-compatible subcone $C_{0}$. Let $V_{0} \subset V$ be a basis of $C_{0}$. Then there are elements $v_{i} \in V-V_{0}$ and $x, y \in C_{0}$ such that $x+v_{i}, y-v_{i} \in C$ and $\phi(x+y)$ $>\phi\left(x+v_{i}\right)+\phi\left(y-v_{i}\right)$. We can define the linear function $f: \operatorname{linspan}\{x+y\} \cap C \rightarrow R$ by $f(\lambda(x+y)):=\lambda \phi(x+y), \lambda \in R$. We have that linspan $\{x+y\} \cap C \subset C_{0}$. By the 1-extension property with respect to $\phi$ of $C$ we can extend $f$ to a function $f_{0}: C_{0} \rightarrow R$ that satisfies $f_{0}(z) \leqslant \phi(z)$ for every $z \in C_{0}$. But for such an extension we have

$$
f_{0}(x)+f_{0}(y)=f(x+y)=\phi(x+y)>\phi\left(x+v_{i}\right)+\phi\left(y-v_{i}\right),
$$

and then

$$
f_{0}(y)-\phi\left(y-v_{i}\right)>\phi\left(x+v_{i}\right)-f_{0}(x)
$$


Let us show that we can not define an extension $\bar{f}$ of the restriction of $f_{0}$ to linspan $\{x, y\}$ $\cap C$ dominated by $\phi$ to the subcone linspan $\left\{x, y, v_{i}\right\} \cap C$, which provides a contradiction since there is a dominated extension to the whole $C$. Suppose that there is such an extension $\bar{f}$ that satisfies $\bar{f}\left(y-v_{i}\right) \leqslant \phi\left(y-v_{i}\right)$. There must be an $\alpha$ with $\bar{f}\left(y-v_{i}\right)$ $=\bar{f}(y)-\alpha$, and then we obtain

$$
\alpha \geqslant \bar{f}(y)-\phi\left(y-v_{i}\right)>\phi\left(x+v_{i}\right)-\bar{f}(x) .
$$

Thus,

$$
\bar{f}\left(x+v_{i}\right)=\bar{f}(x)+\alpha>\bar{f}(x)+\phi\left(x+v_{i}\right)-\bar{f}(x)=\phi\left(x+v_{i}\right),
$$

and hence $\bar{f}$ can not be dominated by $\phi$.

LEMMA 7. Let $C$ be a cancellative cone and let $C_{0}$ be a subcone of $C$. Let $f$ be a linear function $f: C_{0} \rightarrow R$. Then the set $\left\langle C_{0}\right\rangle=\operatorname{linspan}\left\{C_{0}\right\} \cap C$ is a subcone of $C$ and the extension $f_{\left\langle C_{0}\right\rangle}$ of $f$ to $\left\langle C_{0}\right\rangle$ given by $f_{\left\langle C_{0}\right\rangle}(z):=f(x+z)-f(x)$, where $x \in C_{0}$ is an element such that $x+z \in C_{0}$, is a well-defined linear map.

Proof: It is clear that $\left\langle C_{0}\right\rangle$ is a cone, since linspan $\left\{C_{0}\right\}$ and $C$ are cones. Let us show that $f_{\left\langle C_{0}\right\rangle}$ is a well-defined linear map. First we show that for every $z \in\left\langle C_{0}\right\rangle$ there is an element $x \in C_{0}$ such that $x+z \in C_{0}$. Indeed, there are elements $u, v \in C_{0}$ such that $z=u-v$, since $C_{0}$ is a subcone and $z \in \operatorname{linspan}\left\{C_{0}\right\}$, and then $z+v=u \in C_{0}$.

Now, suppose that there are two elements $x, y \in C_{0}$ such that $x+z, y+z \in C_{0}$. Then $f(x+z)+f(y)=f(x+z+y)=f(y+z)+f(x)$ and $f(x+z)-f(x)=f(y+z)-f(y)$. Thus, $f_{\left\langle C_{0}\right)}$ is well-defined. Straightforward calculations show that $f_{\left(C_{0}\right)}:\left\langle C_{0}\right\rangle \rightarrow R$ is a linear function.

The following theorem gives conditions for a function $\phi$ satisfied a dominated extension theorem for every linear function defined on a particular subcone $C_{0}$.

TheOREM 8. Let $C$ be a cancellative cone. Consider a positively homogeneous function $\phi: C \rightarrow R^{+}$. Let $V$ be an algebraic basis such that $C$ is well generated by $V$, and let $C_{0}$ be a $V$-compatible subcone. Then the first of the following statements implies the second one.

(1) The function $\phi$ is $V$-convex with respect to each $V$-compatible subcone that contains $C_{0}$.

(2) For every linear function $f: C_{0} \rightarrow R$ such that

$$
f(x+z)-f(x) \leqslant \phi(z), \quad x, x+z \in C_{0}, z \in C,
$$

there is an extension $\bar{f}$ to $C$ satisfying $\bar{f}(z) \leqslant \phi(z)$ for every $z \in C$.

Proof: Let $f: C_{0} \rightarrow R$ be a linear function satisfying the condition (2). Consider the subcone $\left\langle C_{0}\right\rangle$ of $C$ and the extension $f_{\left(C_{0}\right)}$ of $f$ given by Lemma 7 . The definition of $f_{\left\langle C_{0}\right\rangle}$ makes clear that $f_{\left\langle C_{0}\right\rangle}(z) \leqslant \phi(z)$ for each $z \in\left\langle C_{0}\right\rangle$. Moreover, a direct argument 
shows that $\left\langle C_{0}\right\rangle$ is $V$-compatible if $C$ is well generated by a basis $V=\left\{v_{i}: i \in I\right\}$. Consider a 1-dimensional non trivial extension of $\left\langle C_{0}\right\rangle$ given by an element $v_{i} \in V$ such that

$$
C_{i}=\operatorname{linspan}\left\{v_{i},\left\langle C_{0}\right\rangle\right\} \cap C \neq\left\langle C_{0}\right\rangle .
$$

For each element $z$ of $C_{i}$ there is a unique representation as a sum $z=x+\lambda v_{i}, \lambda \in R$, since $C$ is well generated by $V$. Then we define the extension by mean of the formula $f_{i}(z):=f_{\left\langle C_{0}\right\rangle}(x)+\lambda \alpha$, where $\alpha$ must satisfy

$$
\alpha \leqslant \inf \left\{\phi\left(x+v_{i}\right)-f_{\left\langle C_{0}\right\rangle}(x): x \in\left\langle C_{0}\right\rangle, x+v_{i} \in C_{i}\right\}=\alpha_{1}
$$

and

$$
\alpha \geqslant \sup \left\{f_{\left(C_{0}\right)}(x)-\phi\left(x-v_{i}\right): x \in\left\langle C_{0}\right\rangle, x-v_{i} \in C_{i}\right\}=\alpha_{2}
$$

Given an $\varepsilon>0$, we can find $x_{1}, x_{2} \in\left\langle C_{0}\right\rangle$ such that

$$
\phi\left(x_{1}+v_{i}\right)-f_{\left(C_{0}\right)}\left(x_{1}\right)<\alpha_{1}+\varepsilon
$$

and

$$
\alpha_{2}-\varepsilon<f_{\left(C_{0}\right)}\left(x_{2}\right)-\phi\left(x_{2}-v_{i}\right) .
$$

The following inequalities are consequences of the $V$-convexity of $\phi$ and show that there exists such an $\alpha$, since

$$
f_{\left(C_{0}\right\rangle}\left(x_{1}\right)+f_{\left\langle C_{0}\right\rangle}\left(x_{2}\right)=f_{\left(C_{0}\right)}\left(x_{1}+x_{2}\right) \leqslant \phi\left(x_{1}+x_{2}\right) \leqslant \phi\left(x_{1}+v_{i}\right)+\phi\left(x_{2}-v_{i}\right),
$$

and then

$$
\alpha_{2}-\varepsilon<f_{\left\langle C_{0}\right\rangle}\left(x_{2}\right)-\phi\left(x_{2}-v_{i}\right) \leqslant \phi\left(x_{1}+v_{i}\right)-f_{\left\langle C_{0}\right\rangle}\left(x_{1}\right)<\alpha_{1}+\varepsilon .
$$

Now let us show that $f_{i}(z) \leqslant \phi(z)$ for every $z$ of $C_{i}$. If $z=x+\lambda v_{i}$, where $\lambda \geqslant 0$, we obtain

$$
f_{i}(z)=f_{\left\langle C_{0}\right\rangle}(x)+\lambda \alpha \leqslant f_{\left\langle C_{0}\right\rangle}(x)+\lambda\left(\phi\left(\frac{x}{\lambda}+v_{i}\right)-f_{\left\langle C_{0}\right\rangle}\left(\frac{x}{\lambda}\right)\right) \leqslant \phi\left(x+\lambda v_{i}\right) .
$$

On the other hand, if $z=x-\mu v_{i}$ for $\mu \geqslant 0$,

$$
f_{i}(z)=f_{\left\langle C_{0}\right\rangle}(x)-\mu \alpha \leqslant f_{\left(C_{0}\right\rangle}(x)-\mu\left(f_{\left(C_{0}\right)}\left(\frac{x}{\mu}\right)-\phi\left(\frac{x}{\mu}-v_{i}\right)\right) \leqslant \phi\left(x-\mu v_{i}\right) .
$$

We have shown that there is a non trivial extension of the linear function $f_{\left(C_{0}\right)}$ to $C_{i}$ induced by the element $v_{i} \in V$. Since $C_{0}$ is $V$-compatible, there is a subset $J$ of $I$ such that linspan $\left\{v_{i}: i \in J\right\} \cap C=\left\langle C_{0}\right\rangle$. Consider the set $K=I-J$. If $L \subset K$, we define the subcone $C_{L}$ of $C$ given by $C_{L}=\operatorname{linspan}\{L \cup J\} \cap C$. Note that $C_{0} \subset\left\langle C_{0}\right\rangle \subset C_{L}$ and $\left\langle C_{L}\right\rangle=C_{L}$ for every $L \subset K$. Take the family of subcones of $C$ given by $\mathcal{F}=\left\{C_{L}: L \subset K\right\}$ and define the family of extensions

$$
\overline{\mathcal{F}}=\left\{\left(C_{L}, f_{L, t}\right): C_{L} \in \mathcal{F}, t \in T\right\}
$$


where $T$ is a collection of index such that for each $L \subset K$ and $t \in T, f_{L, t}: C_{L} \rightarrow R$ extends $f_{\left(C_{0}\right)}$ and $f_{L, t}(z) \leqslant \phi(z)$ for every $z \in C_{L}$. Note that the well generation of $C$ by $V$ implies that for each $L$ and for each $r$ which is not in $L \cup J$, if $z \in \operatorname{linspan}\left\{v_{r}, C_{L}\right\} \cap C$ there is a $\lambda \in R$ and an element $x \in C_{L}$ such that $z=x+\lambda v_{r}$. We can define an order relation in $\overline{\mathcal{F}}$ in the usual way, that is, $\left(C_{L}, f_{L, t}\right) \leqslant\left(C_{H}, f_{H, s}\right)$ if $C_{L} \subset C_{H}$ and $f_{H, s}$ extends $f_{L, t}$. To see that every chain gives an upper bound, it is enough to consider the union of all the elements of the chain and the corresponding natural definition of the extension. Moreover, such a union can also be written as an element of $\mathcal{F}$. Since we have shown that $\overline{\mathcal{F}}$ is non void, we can apply Zorn's Lemma to find a maximal element $\left(C_{M}, f_{M, r}\right)$. The proof finishes with the observation that $C_{M}=C$. If this is not the case, we could find an element $v_{r} \in V$ such that $C_{M} \neq \operatorname{linspan}\left\{v_{r}, C_{M}\right\} \cap C$. The same argument given for the construction of the one dimensional extension in the begining of the proof would give a proper extension of $\left(C_{M}, f_{M, r}\right)$, which contradicts its maximality.

Corollary 9. Let $C$ be a cancellative cone and let $\phi: C \rightarrow R^{+}$be a positively homogeneous function. If $C$ has the 1 -extension property with respect to $\phi$, then for every algebraic basis $V$ such that $C$ is well generated by $V$, for every $V$-compatible subcone $C_{0}$ and for every linear function $f: C_{0} \rightarrow R$ such that

$$
f(x+z)-f(x) \leqslant \phi(z), \quad x, x+z \in C_{0}, z \in C,
$$

there is a dominated extension $\bar{f}$ to $C$, that is, $\bar{f}(z) \leqslant \phi(z)$ for every $z \in C$.

We conclude this paper with an application to the case of linear spaces. If the cone is a linear space it is well generated by any algebraic basis. Note that similar arguments could also be used for cones with the same kind of geometric properties. In this case we obtain, up to a point, an equivalence between $V$-convexity, the existence of dominated extensions for functions defined on subcones and the 1-extension property.

DEFinition 10: Let $E$ be a linear space. We say that a positively homogeneus function $\phi: E \rightarrow R^{+}$is a Hahn-Banach function for $E$ if for every subspace $E_{0}$ of $E$ and each algebraic basis $V$ such that $E_{0}$ is $V$-compatible, then every $V$-compatible subspace $E_{c}$ that contains $E_{0}, \phi$ is $V$-convex with respect to $E_{c}$.

COROLlary 11. Let $E$ be a linear space and let $\phi: E \rightarrow R^{+}$be an homogeneous function. Then the following are equivalent.

(1) $\phi$ is a Hahn-Banach function for $E$.

(2) If $x, y, z \in E$ and $z \neq 0$ is not a linear combination of $x$ and $y$, then

$$
\phi(x+y) \leqslant \phi(x+z)+\phi(y-z) .
$$

(3) For every subcone $C_{0}$ of $E$ and every linear function $f: C_{0} \rightarrow R$ such that

$$
f(x+z)-f(x) \leqslant \phi(z), \quad x, x+z \in C_{0}, z \in E,
$$


there is a linear extension $\bar{f}$ to $E$ dominated by $\phi$.

(4) The space $E$ has the 1-extension property with respect to $\phi$.

Proof: The equivalence between (1) and (2) holds from the fact that each linearly independent subset of a linear space can be used to construct an (algebraic) basis for the space (see [8, Theorem 2.4, Chapter IV]). To prove the equivalence of (1) and (3) it is. enough to note that the existence of a dominated extension for every linear functional defined in a subcone $C_{0}$ of $E$ is a direct consequence of Theorem 8 . On the other hand, (3) implies in particular that $E$ has the 1-extension property with respect to $\phi$. Finally, $(4) \rightarrow(1)$ is a consequence of Theorem 6 .

Following $[4,5,6,7]$, an asymmetric norm (quasi-norm in $[1,3]$ ) on a linear space $E$ is a non negative real valued function $\|\cdot\|$ on $E$ such that for all $x, y \in E$ and $\lambda \in R^{+}$,

(i) $\|x\|=\|-x\|=0 \Leftrightarrow x=0$;

(ii) $\|\lambda x\|=\lambda\|x\| ;$ and

(iii) $\|x+y\| \leqslant\|x\|+\|y\|$.

The pair $(E,\|\cdot\|)$ is then called an asymmetric normed linear space.

Each asymmetric norm $\|\cdot\|$ on $E$ induces in a natural way a topology on $E$ which has as a open neighbourhood basis for each $x \in E$ the sets of the form $\{y \in E:\|y-x\|$ $<r\}, r>0$.

Recent applications show that asymmetric norms contitute an efficient tool in the study of some problems in the theory of computational complexity $([\mathbf{1 0}])$ and in approximation theory ([9]).

Since every asymmetric norm is a positively homogeneous function, we can obtain a Hahn-Banach type theorem as a consequence of Theorem 8 . This will be done with the help of the following result, which can be found in [1].

LEMMA 12. Let $\left(X,\|\cdot\|_{1}\right)$ and $\left(Y,\|\cdot\|_{2}\right)$ be two asymmetric normed linear spaces and let $f: X \rightarrow Y$ be a linear function. Then $f$ is continuous if and only if there is $M>0$ such that $\|f(x)\|_{2} \leqslant M\|x\|_{1}$ for all $x \in X$.

Notice that each asymmetric norm $\|\cdot\|$ defined on a linear space $E$ is a convex function, so it is a Hahn-Banach function on $E$. Hence we obtain the following corollary.

COROLlaRy 13. Let $(E,\|\cdot\|)$ be an asymmetric normed linear space. Then for every subcone $C_{0}$ and every linear function $f: C_{0} \rightarrow R$ such that

$$
f(x+z)-f(x) \leqslant\|z\|
$$

for all $x \in C_{0}$ and $z \in E$ with $x+z \in C_{0}$, there exists a continuous linear extension $\bar{f}$ to $E$.

\section{REFERENCES}

[1] C. Alegre, J. Ferrer and V. Gregori, 'On the Hahn-Banach theorem in certain linear quasi-uniform structures', Acta Math. Hungar. 82 (1999), 315-320. 
[2] B. Fuchssteiner and W. Lusky, Convex cones (North-Holland Publishing Co., Amsterdam, 1981).

[3] J. Ferrer, V. Gregori and A. Alegre, 'Quasi-uniform structures in linear lattices', Rocky Mountain J. Math. 23 (1993), 877-884.

[4] L.M. García Raffi, S. Romaguera and E.A. Sánchez Pérez, 'The bicompletion of an asymmetric normed linear space', Acta Math. Hungar. 97 (2002), 183-191.

[5] L.M. García Raffi, S. Romaguera and E.A. Sánchez Pérez, 'On Hausdorff asymmetric normed linear spaces', Houston J. of Math. (to appear).

[6] L.M. García-Raffi, S. Romaguera and E.A. Sánchez Pérez, 'Extensions of asymmetric norms to linear spaces', Rend. Istit. Mat. Univ. Trieste. 33 (2001), 113-125.

[7] L.M. García-Raffi, S. Romaguera and E.A. Sánchez Pérez, 'Sequence spaces and asymmetric norms in the theory of computational complexity', Math. Comput. Modelling 36 (2002), 1-11.

[8] T.W. Hungerford, Algebra, Graduate Texts in Mathematics 73 (Springer-Verla, Berlin, Heidelberg, New York, 1974).

[9] . S. Romaguera and M. Sanchis, 'Semi-Lipschitz functions and best approximation in quasi-metric spaces', J. Approx. Theory 103 (2000), 292-301.

[10] S. Romaguera and M. Schellekens, 'Quasi-metric properties of complexity spaces', Topology Appl. 98 (1999), 311-322.

[11] W. Roth, 'Hahn-Banach type theorems for locally convex cones', J. Austral. Math. Soc. Ser. A 68 (2000), 104-125.

[12] W. Rudin, Functional analysis (McGraw-Hill Publishing Co., New York, Dusseldorf, Johannesburg, 1973).

[13] R. Tix, 'Some results on Hahn-Banach type theorems for continuous d-cones', Theoret. Comput. Sci. 264 (2001), 205-218.

Escuela de Caminos

Departamento de Matemática Aplicada

Universidad Politécnica de Valencia

46071 Valencia

Spain

e-mail: sromague@mat.upv.es

easancpe@mat.upv.es

ovalero@mat.upv.es 